\title{
Vector mesons production off nuclei at the new planned QCD facility at CERN
}

\section{Sergey Gevorkyan*}

Joint Institute for Nuclear Research, Dubna, Russia

E-mail: gevsejinr.ru

We propose to study the production of light vector mesons $V=\rho, \omega, f(1270), K^{*}(892)$ off nuclei targets using the COMPASS-like detector and the M2 pion, kaon and muon beams at CERN[1]. The scattering amplitude of a vector meson off the nucleon at zero angle, averaged over the nucleon spin is determined by two quantities: $\sigma_{T}^{\prime}=\sigma_{T}\left(1-i \alpha_{T}\right)$ and $\sigma_{L}^{\prime}=\sigma_{T}\left(1-i \alpha_{L}\right)$, where $\sigma_{T(L)}$ is the total cross section for the interaction of a transversely (longitudinally) polarized vector meson with the nucleon and $\alpha_{T(L)}=\operatorname{Re} f_{T(L)}(0) / \operatorname{Im}_{T(L)}(0)$ is the ratio of the real to imaginary part of the corresponding amplitudes at zero angle.

Whereas in the charge exchange reaction $\pi^{-}+p \rightarrow V+n$ with dominating pion exchange vector mesons are produced mainly longitudinally polarized (helicity $\lambda=0$ ) the investigation of their production off nuclei $\pi^{-}+A \rightarrow V+A^{\prime}$ ( $A^{\prime}$ is the nuclear excitation or its break-up products) allows to extract the total cross section of the longitudinally polarized vector meson interaction with nucleon $\sigma_{L}(V N)$, a value of which has not yet been measured.

Such measurements are of a current interest as they are complement to investigations of vector mesons photoproduction off nuclei [2] the experimental realization of which is recently proposed at JLAB [3, 4] to be done using the possibilities of the GlueX detector at Hall D, JLAB.

The obvious advantage of the vector mesons production by hadron beam compared with vector mesons photoproduction is that in the charge exchange process $\pi^{-}\left(K^{-}\right)+A \rightarrow V+A^{\prime}$ the main part of vector mesons is longitudinally polarized unlike vector mesons photoproduction, where due to the s-channel helicity conservation $\rho, \phi$ mesons are produced mainly transversely polarized and only a part of $\omega$ mesons at JLAB energies (due to pion exchange) is longitudinally polarized.

XXVII International Workshop on Deep-Inelastic Scattering and Related Subjects - DIS2019

8-12 April, 2019

Torino, Italy

${ }^{*}$ Speaker. 


\section{Introduction}

The dependence of vector particle interactions off nuclei as a function of the particle's polarization has been known for many years in the case when the constituents of the particle are in the D-wave state. A good example of such dependence is the deuteron interaction with matter [5]. The D-wave component in the deuteron wave function leads to different absorption in matter for transversely and longitudinally polarized deuterons $[6,7]$. There are also predictions that the interaction of mesons with nonzero orbital momentum with nucleons is strongly correlated with the meson polarization $[8,9]$. For the ground-state $\mathrm{S}$-wave vector mesons $(\rho, \omega, \phi)$ the $\mathrm{D}$-wave component in their wave functions can emerge as a result of the Lorentz transformation [10]. The origin of a difference between $\sigma_{T}(V N)$ and $\sigma_{L}(V N)$ explained by the appearance of a D-wave contribution in the vector meson wave function in the infinite momentum frame.

The dependence of $\sigma_{L}(\rho N)$ and $\sigma_{T}(\rho N)$ on invariant energy $W=\sqrt{s}$, calculated in the color dipole model with different parameterizations of vector mesons wave functions [11] is shown on Fig. 1. The impact of the polarization on the total cross section of the vector meson interaction with nucleon is large, leading to the different absorption of vector mesons produced on nuclei depending on their polarization.
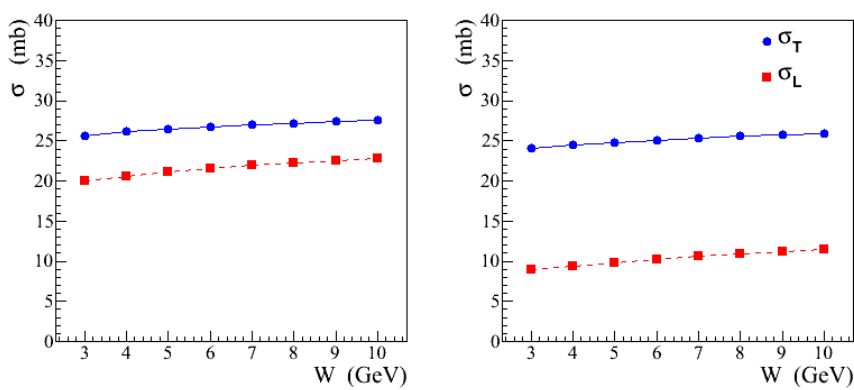

Figure 1: Longitudinal $\sigma_{L}$ and transverse $\sigma_{T} \rho N$ total cross sections as a function of the invariant energy for different parameterizations of the $\rho$ meson wave function: (left) Boosted Gaussian model [12] ; (right) Light-cone relativistic model [13].

\section{Vector mesons production by pions.}

For the best of our knowledge, the only attempt to study the impact of the vector meson polarization on its absorption in nuclei was made many years ago [14] using the charge exchange reaction $\pi^{-}+A \rightarrow \rho^{0}+A^{\prime}$. The incoherent cross section and spin density matrix elements of $\rho$ mesons were measured for different nuclei: $\mathrm{C}, \mathrm{Al}, \mathrm{Cu}, \mathrm{Pb}$.

Due to the dominance of the pion exchange in this process, a large fraction of longitudinally polarized $\rho$ mesons was produced. At the first glance, the experimental data support the assumption that $\sigma_{T}(\rho N) \approx \sigma_{L}(\rho N)$. However, there are strong reasons against such a conclusion. It was shown [15] that, due to the relatively low energy of the primary pion beam $\left(E_{\pi}=3.7 \mathrm{GeV}\right)$ and large decay width of the $\rho$ meson, the significant part of mesons decaying inside the nucleus complicates the interpretation of the experimental data. 
The total cross sections of the $\rho$ and $f(1270)$ mesons with a nucleon were measured at Argonne [16] using the charge exchange process on neon nuclei $\pi^{+}+N e \rightarrow \rho(f)+N e^{\prime}$. Accounting for the possibility of the $\rho$ mesons decay in nuclei $\left(p_{\pi}=3.5 \mathrm{GeV} / c\right)$, the $\rho N$ total cross section is required to be $\sigma(\rho N) \approx 12 \mathrm{mb}$, which would contradicts to the value $\sigma(\rho N) \approx 27 \mathrm{mb}$ obtained from the $\rho$ meson photoproduction on nuclei. From our point of view this difference is a direct result of distinction between $\sigma_{T}(\rho N)$ and $\sigma_{L}(\rho N)$ as in charge exchange process mainly longitudinally polarized $\rho$ mesons are produced, whereas in the photoproduction $\rho$ mesons are transversely polarized due to s-channel helicity conservation.

Taking into account that the vector meson decay mean free path in the nucleus grows with energy $l=\frac{p}{m_{V} \Gamma_{V}}$, the vector mesons produced by pion and kaon beams with energies of tens $\mathrm{GeV}$ available at the M2 beam line, would help to determine uniquely the value of longitudinal $V N$ total cross section $\sigma_{L}(V N)$, while the value of the total cross section for transversely polarized vector mesons $\sigma_{T}(V N)$ is known from their photoproduction on nuclei [17] and can be cross-checked at COMPASS energies. The COMPASS experiment already has some high-statistics data collected with $190 \mathrm{GeV} / c$ negative hadron (mainly pion) beam of high intensity and the set of nuclear targets from $H$ to $P b$ (mainly hydrogen and nickel) collected in 2008, 2009 and 2012. These data can be used for preliminary studies and setup optimizations.

\section{Production of vector mesons $K^{*}(892)$ by kaon beam.}

There are some plans to produce the RF-separated kaon beam for future measurements at the COMPASS experimental area. If so it is interesting to measure the charge exchange process $K^{ \pm}+A \rightarrow K^{*}+A^{\prime}$ to get information on $K^{*}$ absorption in nuclear matter, depending on vector meson polarization. Recently ALICE collaboration presented the preliminary data [18] on $K^{*}$ production in peripheral nucleus-nucleus collisions, which show that vector mesons polarization unlike their production in pp collisions depends on their transverse momenta and centrality.

In the left side of Fig. 2 the spin density matrix element $\rho_{00}$ of $K^{*}$ produced in pp collisions is depicted. The value $\rho_{00}=1 / 3$ corresponds to production of unpolarized vector mesons. On the other hand as is shown at the right part of Fig. 2 in lead-lead collisions at moderate transverse momenta the yield of longitudinally polarized $K^{*}$ are suppressed. Thus the investigation of $K^{*}$ production off nuclei becomes a topical issue.

Feasibility of the proposed measurements with kaon beam could also be tested with available COMPASSI data since the negative hadron beam used by COMPASS contains $\sim 3 \%$ of kaons. 

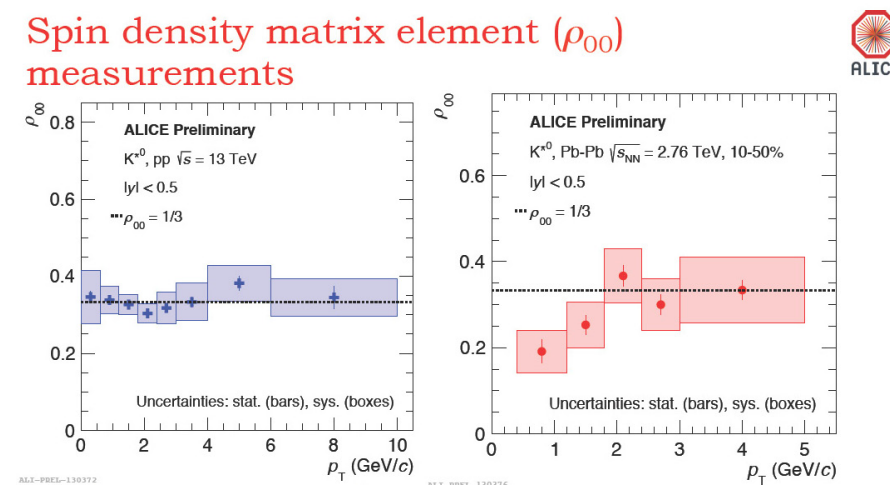

Figure 2: Left: The dependence of $\rho_{00}$ on the transverse momenta of $K^{* 0}$ in the reaction $p+p \rightarrow K^{* 0}+X$; Right: The same dependence in lead-lead collisions $P b+P b \rightarrow K^{* 0}+X$

\section{Vector mesons production by muon beam.}

The knowledge of the cross section $\sigma_{L}(V N)$ is important for interpreting the color screening effect in the vector meson leptoproduction [11]. The idea of the color transparency (CT) is that a hadron produced in certain hard-scattering processes has a smaller probability to interact in the nuclear matter due to its smaller size compared to the physical hadron. As a result the color transparency increases the nuclear transparency defined as $\operatorname{Tr}=\frac{\sigma_{A}}{A \sigma_{N}}$, where $\sigma_{A}$ and $\sigma_{N}$ are vector mesons production cross sections off nuclei and nucleon, respectively.

On the other hand, the nuclear transparency depends on the values of $\sigma_{L}(V N)$ and $\sigma_{T}(V N)$. Fig. 3 represents the dependence of the nuclear transparency in the $\rho$ meson electroproduction as a function of the virtuality of the photon $Q^{2}$. The $\rho$ meson absorption in nuclei decreases with the increase of the $Q^{2}$, which is accounted for the CT effect. At the same time, the fraction of longitudinally polarized vector mesons also rises at large $Q^{2}$ as shown on the right plot of Fig. 3. If one assumes that $\sigma_{T}(V N) \gg \sigma_{L}(V N)$, the effect of the absorption weakening at large $Q^{2}$ cannot be entirely described by the CT. Differences between interaction of longitudinally and transversely polarized vector mesons with nucleon should be considered.

In this way, defining $\sigma_{L}(V N)$ with the help of vector mesons production by hadronic beams the measurement of vector mesons production off nuclei by muons allows one to separate the effect of CT from the absorption weakening due to different absorption of transverse and longitudinally polarized vector mesons in matter.

The interesting possibility, which allows the COMPASS-like facility, is the production of $\omega$ mesons off nucleon and nuclei by muons with the aim of the investigation of the ratio $R=$ $\frac{\sigma\left(\gamma_{L} N \rightarrow \omega N\right)}{\sigma\left(\gamma_{T} N \rightarrow \omega N\right)}$ at moderate $Q^{2}$. While in the case of $\rho, \varphi$ production, this ratio grows with $Q^{2}$ (see right site of Fig. 3) in accordance with gauge invariance, in the case of $\omega$ production by virtual photons this ratio is much less and almost independent of photon virtuality [21](see Fig. 3). This data obtained by HERMES collaboration at $Q^{2}>1(\mathrm{GeV} / \mathrm{c})^{2}$ and energy $3 \mathrm{GeV}<W<6.3 \mathrm{GeV}$ and such behavior has been explained [22] by huge contribution of unnatural parity exchange in transition amplitude $\gamma_{T}^{*} \rightarrow \omega_{T}$. The measurement of $\omega$ photoproduction at smaller photon virtuality $Q^{2}<1(\mathrm{GeV} / \mathrm{c})^{2}$ and higher energies using the COMPASS-like possibilities leads to better 

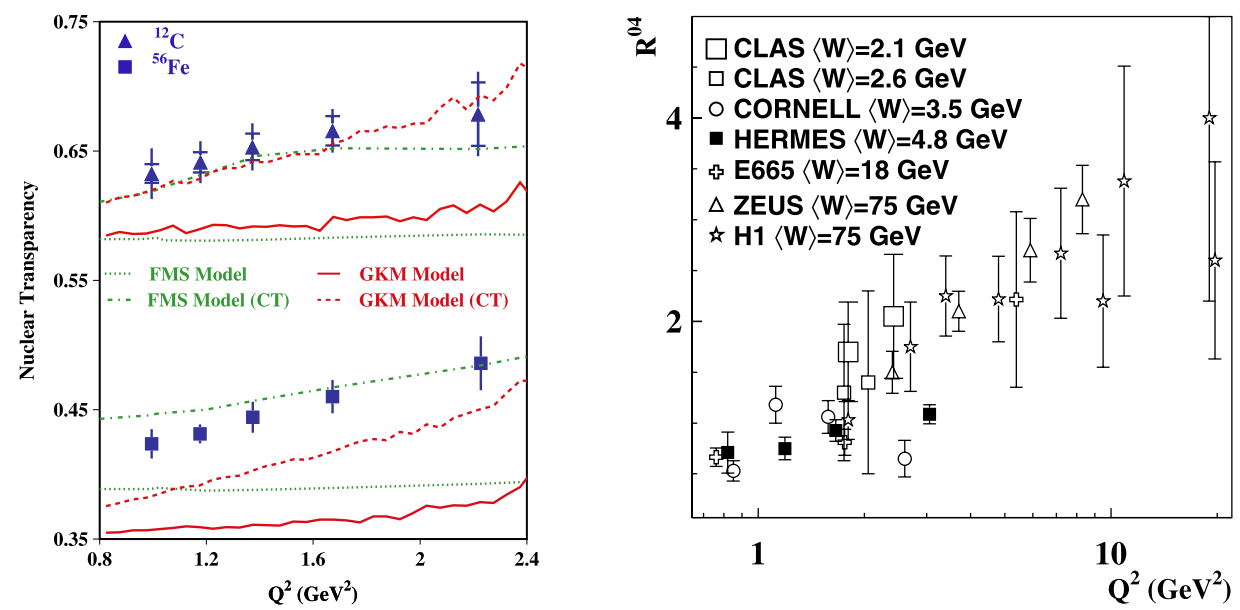

Figure 3: Left: Nuclear transparency of $\rho^{0}$ electroproduction as a function of $Q^{2}$ in different models compared to the experimental data from CLAS [19]. Right: $Q^{2}$ dependence of the ratio of the longitudinal-totransverse cross sections $R=\frac{\sigma\left(\gamma_{L} N \rightarrow \rho N\right)}{\sigma\left(\gamma_{T} N \rightarrow \rho N\right)}$ for exclusive $\rho^{0}$ production on proton [20].

insight in a mechanism of vector mesons production. As for the $\omega$ meson production by muons off nuclei targets such investigations would be a good complement to the experiment on $\omega$ mesons production by real photons planned [4] at JLAB. It is important to note that COMPASS already has experience with muoproduction of $\rho^{0}$ [23] and $\omega$ [24]. Large set of data already collected by COMPASS with low-A polarized targets, hydrogen and nickel targets could help to optimize such kind of measurements.

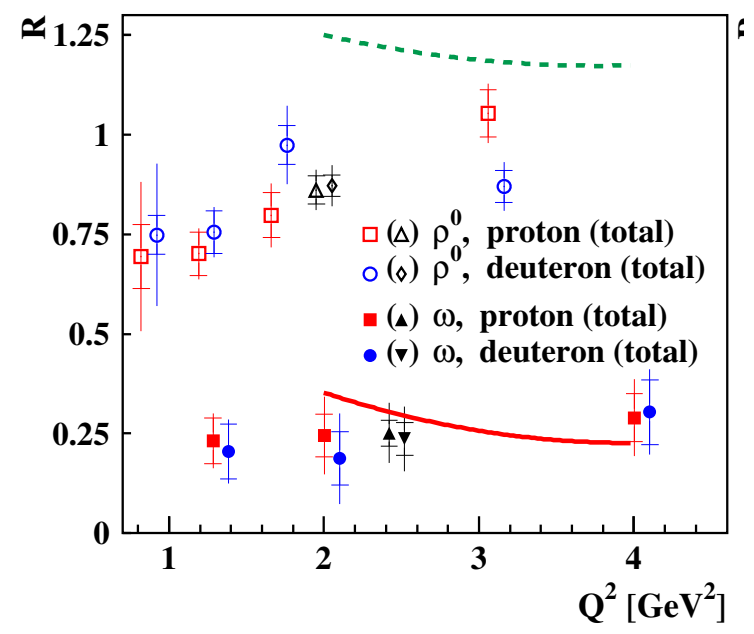

Figure 4: The $Q^{2}$ dependence of the longitudinal to transverse virtual photon cross section ratio $R=$ $\frac{\sigma\left(\gamma_{L} p \rightarrow V p\right)}{\sigma\left(\gamma_{T} N \rightarrow V N\right)}$ for exclusive $\omega$ and $\rho$ electroproduction [21]. 


\section{Acknowledgements}

I am grateful to my colleagues A. V. Guskov and I. A. Savin for the interest and support.

\section{References}

[1] COMPASS++/AMBER, Letter of Intend: A new QCD facility at the M2 beam line at the CERN SPS [hep-ex/1808.00848].

[2] E. Chudakov, S. Gevorkyan, A. Somov, Phys. Rev. C93 (2016) 015203.

[3] E. Chudakov, S. Gevorkyan, A. Somov, Letter of Intend: Study of the $\omega$ mesons photoproduction off nuclei with the GlueX detector JLAB, PAC-43 (2015).

[4] GlueX collaboration, Photoproduction of vector mesons on nuclei with GlueX Proposal PR 12-17-10, PAC-45 (2017).

[5] V. Franco, R. Glauber, Phys. Rev. Lett. 22 (1969) 370.

[6] L. Azhgirey et al., Particles and Nuclei, Letters 5 (2008) 728.

[7] H. Seyfarth et al., Phys. Rev. Lett. 104, (2010) 222501.

[8] L. Gerland et al., Phys. Rev. Lett. 81 (1998) 762.

[9] I.P. Ivanov, N.N. Nikolaev, A.A. Savin, Physics of Particles and Nuclei 37 (2006) 5.

[10] W. Jaus, Phys. Rev. D44 (1991) 2851.

[11] S.R. Gevorkyan, EPJ Web of Conferences 138 (2017) 08004; Jour. of Phys. 678 (2016) 01203.

[12] B. Kopeliovich, J. Nemchik, I. Schmidt, Phys. Rev. C76 (2007) 015205.

[13] J. R. Forshaw, R. Sandapen, JHEP 11 (2010) 037.

[14] A. V. Arefyev et al., Sov. J. Nucl. Phys. 19 (1974) 304; 27 (1978) 85.

[15] A. S. Pak, A. V. Tarasov, Yad. Phys. 22 (1975) 91.

[16] B. Chaudhary et al., Nucl. Phys. B67 (1973) 333.

[17] T. Bauer, R. Spital, D. Yennie, F. Pipkin, Rev. of Mod. Phys. 50 (1978) 261.

[18] B. Mohanty, Measurements of spin alignment of vector mesons and global polarization of hyperons with ALICE at the LHC [nucl-ex/1711.02018].

[19] L. El Fassi et al., Phys. Lett. B 712 (2012) 325.

[20] A. Airapetian et al., Eur. Phys. J. C 64 (2009) 659.

[21] A.Airapetian et al., Eur. Phys. J. C74 (2014) 3110.

[22] S. V. Goloskokov, P. Kroll, Eur. Phys. J. C74 (2014) 2725.

[23] C. Adolph et al., Phys. Lett. B731 (2014) 19.

[24] C. Adolph et al., Nucl. Phys. B915 (2017) 454. 